\title{
The association of findings on brain computed tomography with neurologic outcomes following extracorporeal cardiopulmonary resuscitation
}

Jeong-Am Ryu', Chi Ryang Chung ${ }^{1}$, Yang Hyun Cho ${ }^{2}$, Kiick Sung ${ }^{2}$, Gee Young Suh ${ }^{1,3}$, Taek Kyu Park ${ }^{4}$, Young Bin Song ${ }^{4}$, Joo-Yong Hahn ${ }^{4}$, Jin-Ho Choi ${ }^{4}$, Hyeon-Cheol Gwon ${ }^{4}$, Seung-Hyuk Choi ${ }^{4}$ and Jeong Hoon Yang ${ }^{1,4^{*}}$

\begin{abstract}
Background: Limited data are available on imaging predictors of neurological outcomes after extracorporeal cardiopulmonary resuscitation (ECPR). We investigated the association of initial brain computed tomography (CT) findings with neurological outcomes following ECPR.

Methods: Between February 2005 and December 2015, a total of 42 patients who underwent brain CT scans within $48 \mathrm{~h}$ after ECPR were analyzed. Loss of the boundary between gray matter and white matter (LOB) or cortical sulcal effacement (SE), gray-to-white matter ratio (GWR), and optic nerve sheath diameter (ONSD) were measured on initial brain CT. The primary outcome was the Cerebral Performance Categories (CPC) scale at discharge.

Results: Of the 42 adult ECPR patients, 23 (54.8\%) patients survived to discharge and 19 (45.2\%) patients had good neurological outcomes (CPC 1 and 2). The area under the curve (AUC) of GWR in the basal ganglia (GWR-BG) was 0 . 792 (95\% confidence interval $(C l), 0.639-0.901, p=0.001$ ). ONSD (AUC 0.745; 95\% Cl, $0.587-0.867, p=0.007$ ) was 5. 57 (interquartile range (IQR) $5.14-5.98$ ) $\mathrm{mm}$ in the good neurological outcome group versus 6.07 (IQR $5.71-6.64$ ) $\mathrm{mm}$ in the poor outcome group. LOB or SE were more often detected in the poor neurological outcome group (AUC $0.817 ; 95 \% \mathrm{Cl}, 0.682-0.952, p<0.001$ ). The predictive performance of poor neurological outcomes of a composite of GWR-BG, ONSD, and LOB/SE was significantly improved (AUC 0.904; 95\% Cl, 0.773-0.973) compared to when each brain CT marker was considered separately (GWR-BG, $p=0.048$; ONSD, $p=0.026$; LOB/SE, $p=0.028$ ).
\end{abstract}

Conclusions: GWR, ONSD, and LOB/SE on initial brain CT scans are associated with neurological prognosis in patients who underwent ECPR. The new risk prediction model, which uses a composite of GWR, ONCD, and LOB/ $\mathrm{SE}$, could provide better information on neurologic outcomes in patients underwent ECPR.

Keywords: Brain computed tomography, Cardiopulmonary resuscitation, Extracorporeal membrane oxygenation

\footnotetext{
*Correspondence: jhysmc@gmail.com

'Department of Critical Care Medicine, Samsung Medical Center,

Sungkyunkwan University School of Medicine, 81 Irwon-ro, Gangnam-gu,

Seoul, Republic of Korea

${ }^{4}$ Division of Cardiology, Department of Medicine, Samsung Medical Center,

Sungkyunkwan University School of Medicine, 81 Irwon-ro, Gangnam-gu,

Seoul, Republic of Korea

Full list of author information is available at the end of the article
} 


\section{Background}

Unconscious patients admitted to intensive care units (ICUs) after cardiac arrest are at a high risk of death, and development of neurologic deficits is common among those who survive [1]. In particular, neurological outcomes have been investigated more in patients with stable vital signs and reversible causes of arrest after a return of spontaneous circulation (ROSC). In the setting of conventional cardiopulmonary resuscitation (CPR), several predictors of neurological outcome have been reported such as physical examination of brainstem reflexes, various serum markers, and electrophysiologic study results [2-4]. Additionally, brain imaging may be helpful for predicting neurological outcomes after cardiac arrest [5-11]. Recently, extracorporeal cardiopulmonary resuscitation (ECPR) has been increasingly utilized to supply oxygenated blood and hemodynamic support in the absence of spontaneous cardiac circulation. Similar to studies on conventional CPR, there have been several studies on neurological prognosis in patients receiving ECPR [12-17]. However, there are limited data available on prognostic imaging markers of neurological outcomes after ECPR [18]. Brain computed tomography (CT) is simple, cost-effective, and easily implemented after ECPR [5]. Therefore, we investigated the association of initial brain CT findings with neurological outcomes following ECPR

\section{Methods}

\section{Study population}

This was a retrospective, single-center, observational study of adult patients who underwent ECPR during hospitalization at the Samsung Medical Center between February 2005 and December 2015. This study was approved by the Institutional Review Board of the Samsung Medical Center. The requirement for informed consent was waived due to the retrospective nature of the study.

We included patients who underwent ECPR during the study period, and who were unconscious (a score of $<9$ on the Glasgow Coma Scale on which scores range from 3 to 15 , with lower scores indicating reduced levels of consciousness) on admission to the hospital after cardiac arrest and who were given a brain CT scan within $48 \mathrm{~h}$ after ECPR. Of these patients, we excluded patients under 18 years of age, patients with malignancy whose expected life span was less than 1 year, patients with insufficient medical records, and patients with a history of head trauma, neurosurgery, or a chronic neurological abnormality on ICU admission. A total of 257 patients underwent ECPR between February 2005 and December 2015. Of these, 53 patients underwent brain CT scans within $48 \mathrm{~h}$ after ECPR. Although a brain CT scan was performed after ECPR, a total of 11 patients were excluded from this study (Fig. 1). Finally, a total of 42

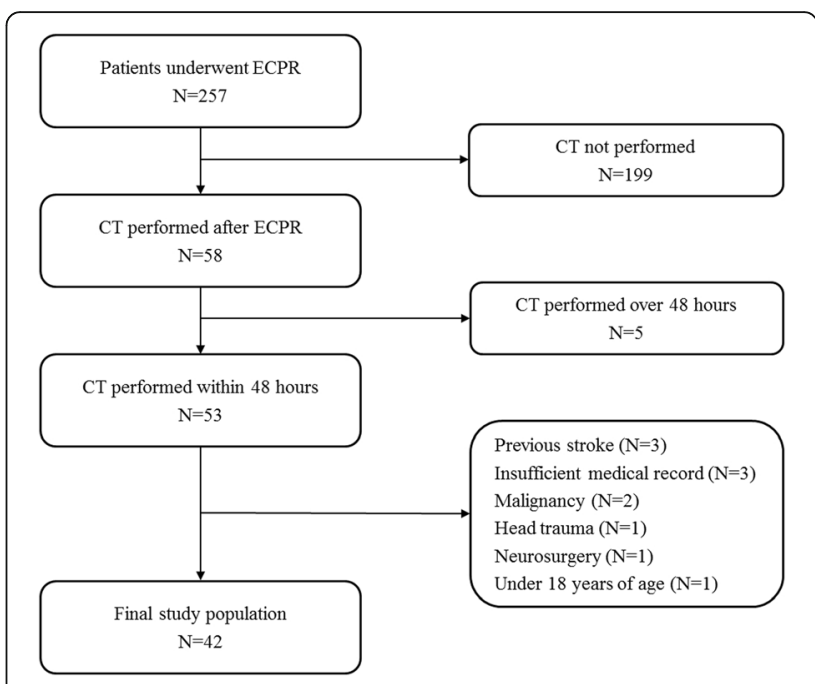

Fig. 1 Study flow chart. CT computed tomography, ECPR extracorporeal cardiopulmonary resuscitation

patients with cardiac arrest who were rescued by venoarterial extracorporeal membrane oxygenation (ECMO) were analyzed in this study.

\section{Definitions and outcomes}

ECPR was defined as both a successful veno-arterial ECMO implantation and pump-on with cardiac massage during the index procedure in patients with cardiac arrest in this study. Actually, when ROSC occurs during ECMO cannulation, the practitioner does not remove the already inserted cannula and does not stop the process of ECMO pump-on $[14,19]$. ECMO pump-on was defined as the status that chest compression was stopped following successful ECMO implantation and activation. At this time, the ECMO flow was gradually increased until respiratory and hemodynamic statuses were stable. In this study, CPR duration was defined as the total time from onset to halting of chest compression. ROSC was defined for all rhythms as restoration of spontaneous rhythm that resulted in more than an occasional gasp, fleeting palpated pulse, or arterial waveform. CPR to ECMO pump-on time was defined as the time from initiation of chest compressions to the time at which the ECMO pump was turned on. Cardiac cause of arrest was presumed to be of cardiac origin unless it was known or likely to have been caused by trauma, submersion, hypothermia, drug overdose, asphyxia, exsanguination, or any other non-cardiac cause including intracranial hemorrhage, and terminal malignancy [20]. Acute coronary syndrome was diagnosed on the basis of electrocardiogram with typical symptoms, cardiac enzyme elevation, and coronary angiographic findings. The primary outcome was neurological status upon hospital discharge, as assessed with the Glasgow-Pittsburgh Cerebral Performance Categories (CPC) scale (1 to 5) 
[21] - CPC 1 and 2 were classified as good neurological outcomes, while CPC 3,4 , and 5 were considered poor neurological outcomes. We thoroughly reviewed medical records, and patients were graded on the CPC scale by two independent neurologists. Targeted temperature management was performed with surface cooling devices. We used a commercial temperature regulation system consisting of a hydrogel pad (Arctic Sun; ${ }^{\circ}$ Medivance Corp, Louisville, CO, USA). Targeted temperature management was determined by intensivists in each ICU. Surface cooling and the degree of targeted temperature were determined by each intensivist in the ICU according to the SMC therapeutic hypothermia protocol [22]. If there were no particular problems, moderate therapeutic hypothermia $\left(32-34{ }^{\circ} \mathrm{C}\right)$ was maintained. However, mild therapeutic hypothermia $\left(35-36{ }^{\circ} \mathrm{C}\right)$ was performed if the patient had hemodyamic instability and bleeding complications during ECMO, or any complications after moderate therapeutic hypothermia. All of the recorded brain CT scans were taken within $48 \mathrm{~h}$ after ROSC in this study. After successful ECPR, a brain CT scan was not performed in patients who had a rapid recovery of mentality and neurological deficits. If not, a brain CT scan was performed to identify whether control of increased intracranial pressure was needed and to exclude intracranial hemorrhage before therapeutic hypothermia by an intensivist. A 64-channel scanner (LightSpeed VCT; GE Healthcare, Milwaukee, Wisconsin, USA) was used for all of the CT studies with a 5-mm slice width. Regions of interest (ROI) were identified by two independent neurologists. Investigators blinded to clinical information opened the CT scans for each patient using commercial image-viewing software (Centricity RA1000 PACS Viewer; GE Healthcare). The images were adjusted to the 'brain' window (window width 90 and window level 30) and comparable brain slices were identified at the level of the basal ganglia (BG) and two levels of the superior cortex, as described previously $[5,9,10]$. Circular regions of measurement $(0.1-$
$0.15 \mathrm{~cm}^{2}$ ) were placed over these ROI, and the average attenuation in Hounsfield units was recorded. At the BG level, the values were recorded bilaterally for the caudate nucleus $(\mathrm{CN})$, putamen $(\mathrm{PU})$, corpus callosum $(\mathrm{CC})$, and posterior limb of the internal capsule (PIC) (Fig. 2). The gray-to-white matter ratio (GWR) in the BG (GWR-BG) was calculated according to a previously reported method as GWR-BG $=(\mathrm{CN}+\mathrm{PU}) /(\mathrm{CC}+\mathrm{PIC})[5,9,10]$. We recorded values bilaterally for the medial cortex and medial white matter at the level of the centrum semiovale (MC1 and MWM1, respectively) and high convexity areas (MC2 and MWM2, respectively). The GWR in the cortex was calculated as GWR-CO $=(\mathrm{MC} 1+\mathrm{MC} 2) \quad / \quad(\mathrm{MWM} 1+$ MWM2) $[5,9,10]$. Average GWR was calculated as the mean of the basal ganglia and cortical GWR (GWR-AV) $=(\mathrm{CN}+\mathrm{PU}+\mathrm{MC} 1+\mathrm{MC} 2) \quad / \quad(\mathrm{CC}+\mathrm{PIC}+\mathrm{MWM} 1+$ MWM2) $[5,9,10]$. The GWR-BG was calculated using a simplified method as reported previously: GWR-simplified $(\mathrm{SI})=\mathrm{PU} / \mathrm{PIC}[5,8,23]$. The ROI were measured and averaged to yield the mean value, and the four GWRs (GWR-BG, GWR-CO, GWR-AV, and GWR-SI) were calculated as described above. Increasing cerebral edema indicates less attenuation of grey matter and a lower GWR. Optic nerve sheath diameter (ONSD) was measured at a distance of $3 \mathrm{~mm}$ behind the eyeball, immediately below the sclera in a perpendicular vector in reference to the linear axis of the nerve (Fig. 3) [11, 24, 25]. The images were changed to the 'chest/abdomen' window (window width 300 and window level 10) and were magnified threefold on the particular image slice which demonstrated the largest diameter of the optic nerve sheath [25]. ONSD was measured from one side of the optic nerve sheath to the other as a section through the center of the optic nerve [24]. The diameters measured for the patient's left and right eyes were averaged to yield a mean value [24]. Loss of the boundary between gray matter and white matter (LOB) was evaluated at the level of the BG on the initial CT scans. The presence of cortical sulcal effacement (SE) was evaluated at the level of the centrum

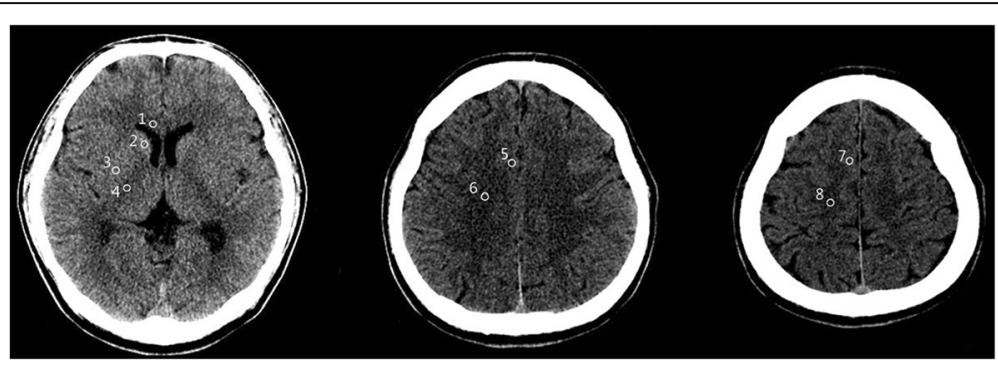

Fig. 2 Brain computed tomography images showing measurements in Hounsfield units at the level of the basal ganglia (left), the centrum semiovale (middle), and the high convexity (right). The circular regions of interest are positioned in the genu of the corpus callosum (1), caudate nucleus (2), putamen (3), and posterior limb of the internal capsule (4) at the level of the basal ganglia and are positioned in the medial cortex $(5,7)$ and medial white matter $(6,8)$ at the centrum semiovale and high convexity levels, respectively 


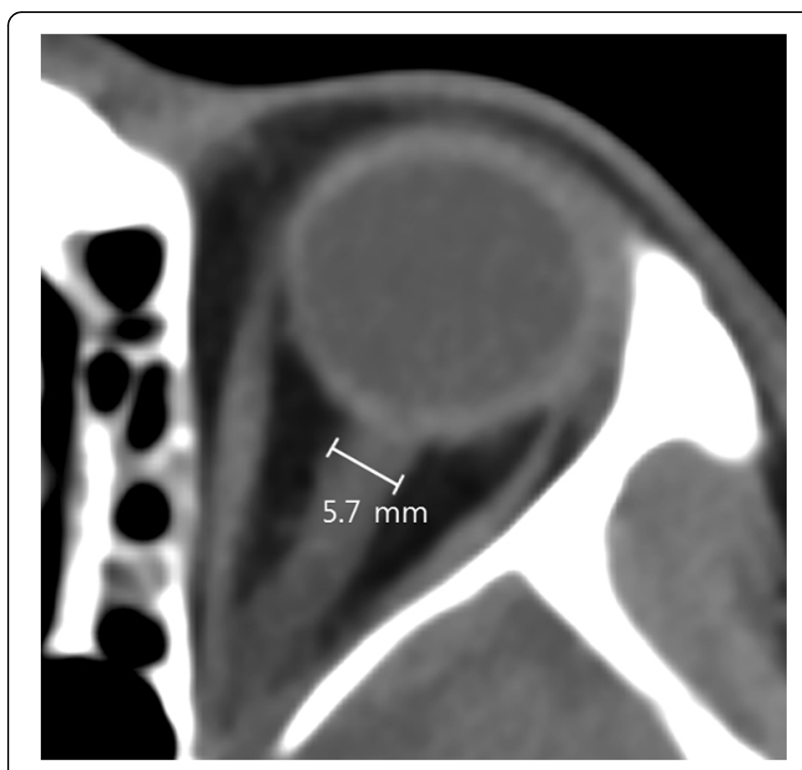

Fig. 3 Measurement of optic nerve sheath diameter on initial brain computed tomography scan

semiovale, and a positive SE sign was defined as SE detectable at this level $[8,23]$.

\section{Procedure}

CPR was led by the CPR team of the hospital, and all facts related to the CPR scene were recorded by bedside nurses according to Utstein-style guidelines [26]. When CPR was performed for more than $10 \mathrm{~min}$ or in the event of unstable vital signs or recurrent cardiac arrest, the institutional rapid response team contacted the oncall ECMO team leader who, along with the CPR leader, assessed the patient and made a decision about whether to institute ECPR. ECPR was performed when a witnessed arrest was confirmed, the arrest persisted despite conventional CPR lasting for more than $10 \mathrm{~min}$, and the event that caused the arrest was considered reversible [18]. Cases in which ECPR was deferred included a short life expectancy ( $<6$ months), terminal malignancy, an unwitnessed collapse, limited physical activity, an unprotected airway, or CPR undertaken for more than $60 \mathrm{~min}$ at the time of initial contact. Age alone did not constitute a contraindication to ECPR [18].

The ECMO team consisted of cardiologists, cardiovascular surgeons, intensivists, special nurses, and perfusionists. Either the Capiox Emergency Bypass System (Terumo, Tokyo, Japan) or the Prolonged Life Support System (Maquet Cardiopulmonary, Hirrlingen, Germany) was used in all cases. A crystalloid solution such as normal saline or balanced solution was used for priming; no patient had blood-primed ECMO. A percutaneous vascular approach was tried initially in all cases using the Seldinger technique, but when percutaneous cannulation failed surgical cut-down exposure was performed [18]. The femoral vessels were the most common sites of vascular access, with the use of 14 to 17 French arterial cannulas and 20 to 24 French venous cannulas [19]. Cardiac compression was stopped once ECMO pump-on was successful during CPR. Anticoagulation was accomplished by a bolus injection of unfractionated heparin, followed by continuous intravenous heparin infusion to maintain an activated clotting time between 150 and $180 \mathrm{~s}$. The initial number of revolutions per minute of the ECMO device was adjusted to achieve an ideal cardiac index greater than $2.2 \mathrm{~L} /$ $\mathrm{min} / \mathrm{m}^{2}$ of body surface area, central mixed venous oxygen saturation above $70 \%$, and a mean arterial pressure above $65 \mathrm{~mm} \mathrm{Hg}$ [19]. Blood pressure was monitored continuously through an arterial catheter, and an artery in the right arm was used for arterial blood gas analysis to estimate cerebral oxygenation. After ECMO, the necessary steps were taken to treat the cause of the arrest, such as percutaneous coronary intervention, coronary artery bypass grafting, heart transplantation, non-coronary cardiopulmonary surgery, or non-cardiopulmonary surgery [19].

\section{Statistical analyses}

All data are presented as medians and interquartile ranges (IQRs) for continuous variables and as numbers (percentages) for categorical variables. Data were compared using the Mann-Whitney $U$ test for continuous variables, and the Chi-square test or Fisher's exact test for categorical variables. The predictive performance of each brain $\mathrm{CT}$ marker was assessed using the area under the curve (AUC) of the receiver operating characteristic (ROC) curves of the sensitivity over 1 - specificity. AUCs were compared using the nonparametric approach published by DeLong et al. [27] for two correlated AUCs. The optimal cut-off values of each brain CT marker for predicting poor neurological outcome were obtained by ROC curve and Youden index [28, 29]. All tests were two-sided, and $p$ values $<0.05$ were considered to indicate statistical significance. Data were analyzed using IBM SPSS statistics version 20 (IBM, Armonk, NY, USA).

\section{Results}

\section{Baseline characteristics}

The median patient age was 51 (range 25-82, IQR 3465) years, and 29 patients (31.0\%) were men. A cardiac cause of arrest was verified in $35(83.3 \%)$ patients. Acute coronary syndrome was the main cause of cardiac arrest in 10 patients $(23.8 \%)$ and 7 patients (16.7\%) had a history of ischemic heart disease. Refractory arrhythmia was verified in 9 patients (21.4\%). Thirty-five patients (83.3\%) experienced cardiac arrest in the hospital and 7 patients (16.7\%) had cardiac arrest in an out-of-hospital setting. The median CPR to ECMO pump-on time was 45 (IQR 27-55) min. Twenty patients (47.6\%) had ROSC (median $7.5 \mathrm{~min}$, range $1-47 \mathrm{~min}$ ). Among these 
patients, only 4 patients had over 20 min ROSC duration and 13 patients had less than 10 min ROSC duration. Even 4 patients with $\mathrm{ROSC}>20 \mathrm{~min}$ had recurrent cardiac arrest before ECMO insertion, and no-flow time was sustained after the last ROSC. The baseline characteristics of ECPR patients are presented in Table 1 and the characteristics of cardiac arrest are given in Table 2. There were no significant differences between the two groups except for a history of diabetes mellitus at baseline and arrest-related characteristics.

\section{Clinical and neurological outcomes}

Among 42 adult cardiac arrest patients who underwent ECPR, successful ECMO weaning was achieved in 29 patients $(69.0 \%)$ and survival to discharge was identified in
23 patients $(54.8 \%)$. Of these 23 survivors, 19 patients (45.2\%) had good neurological outcomes (CPC 1 and 2). The ICU mortality rate was $40.5 \%$ (17 patients). Of the 42 patients, target temperature management was performed in 16 patients (38.1\%) using surface cooling devices.

\section{Brain CT findings}

Although there were no significant differences between the average attenuations in Hounsfield units of each ROI, GWRs differed significantly between the two groups except for GWR-CO. GWRs were higher in the good neurological outcome group (Table 3 ). The ONSD was 5.57 (IQR 5.14-5.98) $\mathrm{mm}$ in the good outcome group versus 6.07 (IQR 5.71-6.64) $\mathrm{mm}$ in the poor

Table 1 Baseline characteristics of comatose patients who underwent brain CT scans after ECPR

\begin{tabular}{|c|c|c|c|c|}
\hline & $\begin{array}{l}\text { Good neurological outcome } \\
(n=19)\end{array}$ & $\begin{array}{l}\text { Poor neurological outcome } \\
(n=23)\end{array}$ & Total $(n=42)$ & $p$ value \\
\hline Age, years (median $(I Q R))$ & $41.0(32.0-70.5)$ & $52.0(43.0-60.5)$ & $51.0(34.0-65.0)$ & 0.503 \\
\hline Gender, male ( $n(\%))$ & $14(73.7)$ & $15(65.2)$ & $29(31.0)$ & 0.739 \\
\hline Body mass index $\left(\mathrm{kg} / \mathrm{m}^{2}\right)$ & $23.1(21.1-25.7)$ & $24.1(22.2-28.5)$ & $23.6(21.5-26.7)$ & 0.105 \\
\hline \multicolumn{5}{|l|}{ Medical history (n (\%)) } \\
\hline Diabetes mellitus & $1(5.3)$ & $10(43.5)$ & $11(26.2)$ & 0.005 \\
\hline Hypertension & $3(15.8)$ & $11(47.8)$ & $14(33.3)$ & 0.048 \\
\hline Malignancy & $3(15.8)$ & $5(21.7)$ & $8(19.0)$ & 0.709 \\
\hline Smoking & $7(36.8)$ & $11(47.8)$ & $18(42.9)$ & 0.474 \\
\hline Previous myocardial infarction & $0(0)$ & $2(8.7)$ & $2(4.8)$ & 0.492 \\
\hline Initial Glasgow Coma Scale (median (IQR)) & $3(3-6)$ & $3(3-4)$ & $3(3-5)$ & 0.518 \\
\hline Target temperature manage by surface cooling device (n (\%)) & $7(36.8)$ & $9(39.1)$ & $16(38.1)$ & 0.879 \\
\hline Interval between ECPR and CT scan ( $n(\%))$ & & & & 0.001 \\
\hline $0-6 h$ & $8(42.1)$ & $11(47.8)$ & $19(45.2)$ & \\
\hline $6-12 h$ & $0(0)$ & $2(8.7)$ & $2(4.8)$ & \\
\hline $12-24 h$ & $10(52.6)$ & $1(4.3)$ & $11(26.2)$ & \\
\hline $24-48 h$ & $1(5.3)$ & $9(39.1)$ & $10(23.8)$ & \\
\hline \multicolumn{5}{|l|}{ Laboratory data on admission (median (IQR)) } \\
\hline Initial lactate (mg/dl) & $83.8(51.4-127.0)$ & $105.4(85.6-136.9)$ & $97.3(68.5-132.4)$ & 0.133 \\
\hline$(\mathrm{mmol} / \mathrm{l})$ & $9.3(5.7-14.1)$ & $11.7(9.5-15.2)$ & $10.8(7.6-14.7)$ & \\
\hline Hemoglobin before ECPR (g/dl) & $13.9(10.5-15.6)$ & $12.0(11.0-14.0)$ & $12.6(10.9-14.6)$ & 0.263 \\
\hline$(\mathrm{mmol} / \mathrm{l})$ & $8.6(6.5-9.7)$ & $7.4(6.8-8.7)$ & $7.8(6.8-9.1)$ & \\
\hline Hemoglobin after ECPR (g/dl) & $10.5(8.8-11.9)$ & $9.9(8.8-11.1)$ & $10.2(8.8-11.5)$ & 0.597 \\
\hline$(\mathrm{mmol} / \mathrm{l})$ & $6.5(5.5-7.4)$ & $6.1(5.5-6.9)$ & $6.3(5.5-7.1)$ & \\
\hline Total bilirubin (mg/dl) & $0.7(0.5-1.2)$ & $1.0(0.5-1.2)$ & $0.8(0.5-1.2)$ & 0.744 \\
\hline$(\mu \mathrm{mol} / \mathrm{l})$ & $12.0(8.6-20.5)$ & $17.1(8.6-20.5)$ & $13.7(8.6-20.5)$ & \\
\hline Blood urea nitrogen (mg/dl) & $16.7(14.7-23.0)$ & $17.2(13.6-21.8)$ & $16.7(14.0-22.4)$ & 0.745 \\
\hline$(\mathrm{mmol} / \mathrm{l})$ & $6.0(5.2-8.2)$ & $6.1(4.9-7.8)$ & $6.0(5.0-8.0)$ & \\
\hline Creatinine (mg/dl) & $1.2(1.0-2.0)$ & $1.3(1.1-1.7)$ & $1.3(1.1-1.8)$ & 0.533 \\
\hline$(\mu \mathrm{mol} / \mathrm{l})$ & $106.1(88.4-176.8)$ & $114.9(97.2-150.3)$ & $114.9(97.2-159.1)$ & \\
\hline
\end{tabular}

$C T$ computed tomography, ECPR extracorporeal cardiopulmonary resuscitation, $I Q R$ interquartile range 
Table 2 Characteristics of cardiac arrest in comatose patients who underwent brain CT scans after ECPR

\begin{tabular}{|c|c|c|c|c|}
\hline & $\begin{array}{l}\text { Good neurological outcome } \\
(n=19)\end{array}$ & $\begin{array}{l}\text { Poor neurological outcome } \\
(n=23)\end{array}$ & Total $(n=42)$ & $p$ value \\
\hline Type of cardiac arrest ( $n(\%))$ & & & & 0.999 \\
\hline Out-of-hospital cardiac arrest & $3(15.8)$ & $4(17.4)$ & $7(16.7)$ & \\
\hline In-hospital cardiac arrest & $16(84.2)$ & 19 (82.6) & $35(83.3)$ & \\
\hline Bystander-witnessed cardiac arrest ( $n(\%))$ & $19(100.0)$ & $23(100.0)$ & $42(100.0)$ & \\
\hline Bystander-performed CPR ( $n(\%))$ & $17(89.5)$ & $23(100.0)$ & $40(95.2)$ & 0.199 \\
\hline First monitored rhythm (n (\%)) & & & & 0.714 \\
\hline Asystole & $2(10.5)$ & $3(13.0)$ & $5(11.9)$ & \\
\hline Pulseless electrical activity & $8(42.1)$ & $11(47.8)$ & $19(45.2)$ & \\
\hline Shockable rhythm (VT or VF) & $8(42.1)$ & $9(39.1)$ & $17(40.5)$ & \\
\hline Defibrillation (n (\%)) & $10(55.6)$ & $13(56.5)$ & $23(56.1)$ & 0.951 \\
\hline ROSC before ECMO insertion $(n(\%))$ & $8(44.4)$ & $12(52.2)$ & $20(48.8)$ & 0.623 \\
\hline CPR to ECMO pump-on time, min (median (IQR)) & $43.5(19.0-55.0)$ & $47.0(33.5-56.0)$ & $45.0(27.0-55.0)$ & 0.430 \\
\hline Location of ECMO insertion (n (\%)) & & & & 0.507 \\
\hline Intensive care unit & $6(31.6)$ & $5(21.7)$ & $11(26.2)$ & \\
\hline Cath room & $3(15.8)$ & $7(30.4)$ & $10(23.8)$ & \\
\hline Emergency room & $10(52.6)$ & $10(43.5)$ & $20(47.6)$ & \\
\hline Operation room & $0(0)$ & $1(4.3)$ & $1(2.4)$ & \\
\hline Cardiac cause of arrest $(n(\%))$ & $17(89.5)$ & $18(78.3)$ & $35(83.3)$ & 0.371 \\
\hline Acute coronary syndrome & $3(15.8)$ & $7(30.4)$ & $10(23.8)$ & \\
\hline STEMI & $1(5.3)$ & $3(13.0)$ & $4(9.5)$ & \\
\hline NSTEMI & $1(5.3)$ & $3(13.0)$ & $4(9.5)$ & \\
\hline Unstable angina & $1(5.3)$ & $1(4.3)$ & $2(4.8)$ & \\
\hline Cardiomyopathy & $1(5.3)$ & $0(0)$ & $1(2.4)$ & \\
\hline Acute aortic syndrome & $0(0)$ & $2(8.7)$ & $2(4.8)$ & \\
\hline Pulmonary thromboembolism & $3(17.6)$ & $3(13.0)$ & $6(14.3)$ & \\
\hline Refractory arrhythmia & $5(26.3)$ & $4(17.4)$ & $9(21.4)$ & \\
\hline Left ventricular wall rupture & $4(21.1)$ & $1(4.3)$ & $5(11.9)$ & \\
\hline Other & $1(5.3)$ & $1(4.3)$ & $2(4.8)$ & \\
\hline
\end{tabular}

$C P R$ cardiopulmonary resuscitation, $C T$ computed tomography, ECMO extracorporeal membrane oxygenation, ECPR extracorporeal cardiopulmonary resuscitation, IQR interquartile range, NSTEMI non-ST segment elevation myocardial infarction, ROSC return of spontaneous circulation, STEMI ST segment elevation myocardial infarction, $V T$ ventricular tachycardia, $V F$ ventricular fibrillation

outcome group $(p=0.007)$. LOB sign and SE sign were more likely to be detected in the poor neurological outcome group than in the good neurological outcome group (both $p<0.001$ ). These CT markers of each outcome are shown in Table 3 . The cut-off value, specificity, and sensitivity of these CT markers for poor outcome are shown in Additional file 1 (Table S1). For the ROC curve analysis for the prediction of poor neurological outcome, GWR-BG had the largest AUC (0.792; 95\% confidence interval (CI), 0.639-0.901) among all the GWRs (Fig. 4a). Although there were no differences between the AUCs of GWR-BG, ONSD, and LOB/SE, the performance of a composite of these markers (AUC 0.904, Brier score 0.123, Goodness-of-fit (HosmerLemeshow) $\left.X^{2}=7.562, p=0.477\right)$ was more associated with poor neurological outcomes than the use of each brain CT marker alone (Fig. 4b). Eight patients (19.0\%) had all three poor markers when poor markers of GWR-BG and ONSD were defined according to cut-off values in Additional file 1 (Table S1) (GWR-BG $<1.23$ and ONSD $>5.86 \mathrm{~mm}$, respectively). All of them had poor neurological outcome (sensitivity $34.8 \%$ and specificity $100 \%)$. The number of patients with two or more poor markers was $17(40.5 \%)$. Of these patients, only one patient had good neurological outcome (sensitivity $69.6 \%$ and specificity $94.7 \%)$.

\section{Discussion}

ECPR has been increasingly utilized to supply oxygenated blood and hemodynamic support in the absence of 
Table 3 Comparisons of the attenuation in regions of interest and of the gray-to-white matter ratio (GWR), optic nerve sheath diameter, and early computed tomography signs between groups

\begin{tabular}{|c|c|c|c|c|c|}
\hline & & & $\begin{array}{l}\text { Good neurological outcome } \\
(n=19)\end{array}$ & $\begin{array}{l}\text { Poor neurological outcome } \\
(n=23)\end{array}$ & $p$ value \\
\hline \multicolumn{6}{|l|}{ Density of regions of interest, $\mathrm{HU}$ (median (IQR)) } \\
\hline \multirow[t]{4}{*}{ Basal ganglia } & Gray matter & $\mathrm{CN}$ & $34.90(32.25-38.10)$ & $32.40(28.20-35.60)$ & 0.071 \\
\hline & & PU & $35.70(33.40-38.05)$ & $33.90(31.25-35.9)$ & 0.129 \\
\hline & White matter & $\mathrm{CC}$ & $27.90(24.20-30.60)$ & $27.40(25.55-29.75)$ & 0.752 \\
\hline & & $\mathrm{PIC}$ & $27.70(25.05-29.20)$ & $27.30(26.15-28.80)$ & 0.950 \\
\hline \multirow[t]{2}{*}{ Centrum semiovale (cortical) } & Gray matter & MC1 & $31.90(28.75-34.30)$ & $30.80(27.65-32.45)$ & 0.306 \\
\hline & White matter & MWM1 & $24.9(22.25-29.55)$ & $25.30(23.40-28.10)$ & 0.667 \\
\hline \multirow[t]{2}{*}{ High convexity area (cortical) } & Gray matter & MC2 & $30.70(28.85-33.80)$ & $29.70(28.25-32.05)$ & 0.596 \\
\hline & White matter & MWM2 & $24.60(21.85-27.05)$ & $24.70(22.00-27.45)$ & 0.527 \\
\hline \multicolumn{6}{|l|}{ GWR (median (IQR)) } \\
\hline GWR-BG (basal ganglia) & & & $1.31(1.25-1.37)$ & $1.21(1.11-1.28)$ & 0.001 \\
\hline GWR-CO (cortical) & & & $1.23(1.17-1.32)$ & $1.20(1.09-1.29)$ & 0.098 \\
\hline GWR-SI (simplified) & & & $1.29(1.25-1.38)$ & $1.21(1.13-1.29)$ & 0.023 \\
\hline GWR-AV (average) & & & $1.29(1.23-1.31)$ & $1.21(1.10-1.26)$ & 0.007 \\
\hline Optic nerve sheath diameter, mm (median (IQR)) & & & $5.57(5.14-5.98)$ & $6.07(5.71-6.64)$ & 0.007 \\
\hline LOB at level of basal ganglia $(n(\%))$ & & & $2(10.5)$ & $15(65.2)$ & $<0.001$ \\
\hline SE at level of centrum semiovale $(n(\%))$ & & & $2(10.5)$ & $15(65.2)$ & $<0.001$ \\
\hline
\end{tabular}

CC corpus callosum, $C N$ caudate nucleus, $H U$ Hounsfield units, $I Q R$ interquartile range, $L O B$ loss of boundary between gray matter and white matter, $M C$ medial cortex, MWM medial white matter, PIC posterior limb of the internal capsule, $P U$ putamen, $S E$ cortical sulcal effacement

spontaneous cardiac circulation. Therefore, ECPR may be considered in settings where it can be rapidly implemented among selected cardiac arrest patients who have not responded to initial conventional CPR [30]. In addition, ECPR had a short-term and long-term survival benefit over conventional CPR in patients with in-hospital cardiac arrest of cardiac origin [14,31]. Similar to studies on conventional CPR, there have been several studies on neurological prognosis in patients receiving ECPR [12-17]. However, limited data are available on prognostic imaging markers of neurological outcomes after ECPR [18].

In the present study, we evaluated whether GWR, ONSD, and LOB/SE as measurable variables on initial brain $\mathrm{CT}$ scans were associated with neurological outcome in patients who underwent ECPR. Although there
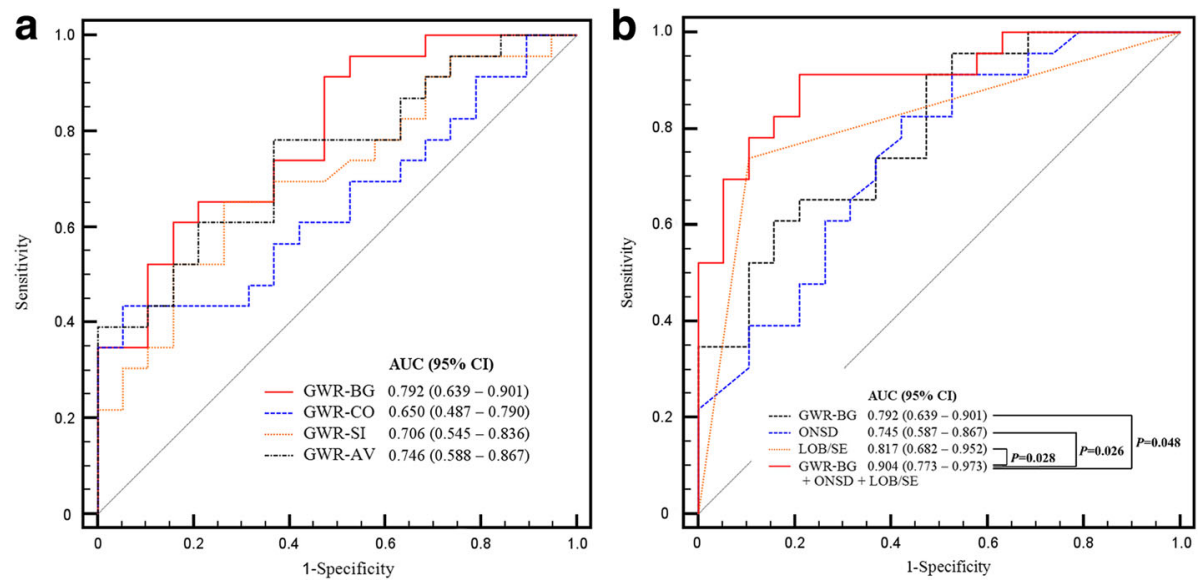

Fig. 4 ROC curves for the prediction of poor outcomes. a ROC curves for the prediction of poor outcomes using the gray-to-white matter ratio of the basal ganglia (GWR-BG), cortex (GWR-CO), simplified (GWR-SI), and average (GWR-AV). b ROC curves for the prediction of poor outcomes using the GWR-BG, optic nerve sheath diameter (ONSD), loss of boundary between gray matter and white matter and cortical sulcal effacement $(L O B / S E)$, and a combination of the three markers. AUC area under the curve, $C l$ confidence interval 
were no significant differences between the average attenuation in Hounsfield units of each ROI, GWRs (except GWR-CO) on initial brain CT were higher in the good neurological outcome group than in the poor outcome group. ONSD on initial brain CT was correlated closely with neurologic outcome and was greater in the poor neurological outcome group. LOB sign and SE sign were more likely to be detected in the poor neurological outcome group than in the good neurological outcome group. GWR, ONSD, and LOB/SE were associated with neurological outcome after ECPR, and these three CT markers had statistically similar predictive power on poor neurological outcome. Furthermore, a composite of GWR-BG, ONSD, and LOB/SE was a significantly greater predictor of poor neurological outcomes than were each brain CT marker alone.

There have been reports on several predictors of neurological outcome after conventional CPR [2]. Absence of brainstem reflex and motor response other than extensor posturing over a period of $72 \mathrm{~h}$ and myoclonic status epilepticus are markers of poor outcomes after cardiac arrest $[2,3]$. Elevated serum S-100B and neuron-specific enolase levels and bilaterally absent somatosensory evoked potentials appear to have prognostic value in predicting a poor outcome [2, 3]. In addition, there have been reports of neuroimaging studies such as brain CT, brain magnetic resonance imaging, and magnetic resonance spectroscopy after conventional CPR [2]. In particular, brain CT scans may be helpful for predicting neurological outcomes after ECPR because brain magnetic resonance imaging is an expensive and time-consuming procedure and cannot be immediately performed because of ECMO equipment in patients receiving ECPR [5]. Sedation may confound outcome prediction in survivors of cardiac arrest [32]. Sedative medications are commonly used in comatose CPR survivors for $72 \mathrm{~h}$ and are an important confounder [32]. Some clinical signs and examinations such as a motor response to noxious stimuli, corneal reflex, caloric testing, and electroencephalography may also be confounded by sedation $[2,32]$. Therefore, brain CT may be more helpful for predicting neurological outcome in ECPR patients under sedation. However, there are limited data on prognostic markers of brain CT for neurological outcomes after ECPR $[13,18]$.

Some signs on brain CT have been associated with ischemic cerebral insult and poor neurological outcome $[5,7-11]$. The LOB and SE signs are associated with ischemic brain damage and poor outcome after cardiac arrest [8]. These signs were also associated with poor neurological outcomes after ECPR in this study. However, identification of these signs may be subject to the abilities of each investigator, and these signs are not quantifiable. GWR was associated with neurological outcome in patients receiving both conventional CPR and ECPR [5, 7-11]. GWR may be explained by the quantitative association of cerebral edema following hypoxic injury with increased intracranial pressure. Similar to other studies, GWRs were significantly different and the attenuation in Hounsfield units in the ROIs were not statistically different between the two groups in this study $[5,9,10]$. In previous studies, average GWR was a better predictor of neurologic outcome than the GWRs of single regions such as BG or the cerebellum alone [5, 9-11]. However, GWR-BG had the largest AUC for prediction of poor neurological outcome among the GWRs in this study. The predictive power of GWR-AV may have been decreased because GWR-CO had little association with poor neurological outcomes in this study. GWRs may be a useful predictor of neurological outcome after ECPR, although the GWR cut-off values were different in different studies $[5,9,10]$.

Measurement of ONSD has been proposed as an alternative method for the detection of increased intracranial pressure [11]. The optic nerve is surrounded by cerebrospinal fluid because it is a part of the central nervous system. Therefore, increased intracranial pressure will be transmitted through the subarachnoid space surrounding the optic nerve within the nerve sheath, especially the retrobulbar segment, unless circulation of cerebrospinal fluid is not blocked [11]. ONSD on initial brain CT may be correlated with neurologic outcomes after cardiac arrest $[11,24,25]$. Simultaneous measurement of ONSD on initial CT and intracranial pressure were correlated, and ONSD was indicative of intracranial hypertension in patients with severe traumatic brain injury. ONSD may be a good predictive marker associated with intracranial pressure [25]. Although ONSD is easy to measure on a brain CT scan, it may sometimes be difficult to measure ONSD precisely because of an inappropriate thickness of a CT section or artifacts on brain CT. In addition, similar to GWR, the cut-off values of ONSD differ between studies $[11,24,25]$. We developed a new predictive model for neurological outcomes to overcome a weakness caused by the variation in cut-off values of GWR and ONSD, and a composite of GWR, LOB/SE, and ONSD was a powerful predictor of neurological outcomes compared with each parameter alone. In real-world practice, combined CT parameters could provide improved information on early neurological prognosis in patients undergoing ECPR.

This study had several limitations. First, it was a retrospective review of medical records. Thus, the CPC scale was also retrospectively determined based on medical records. Second, we used a small heterogeneous group of subjects over a large period of time from a single institution. Over this time frame there has also been a significant change in post-arrest management which may affect patients' outcomes over the study period. Third, the nonrandomized nature of the registry data could 
have resulted in selection bias. Although brain CT scans were performed within $48 \mathrm{~h}$ following ECPR, a major limitation of the study might be that the $\mathrm{CT}$ scans are performed in different time settings. However, in this study, AUC of a composite of three markers (GWR-BG + ONSD + LOB/SE) on brain CT within $15.2 \mathrm{~h}$ (median time) was not significantly different from that after $15.2 \mathrm{~h}(0.886$ (95\% CI, 0.749-0.963) vs. 0.943 (95\% CI, $0.825-0.991) ; p=0.448)$. One-third of patients received targeted temperature management. Hypothermia can cause coagulopathy, so therapeutic hypothermia was not attempted if patients had substantial bleeding after ECMO insertion. Therefore, surface cooling devices such as Arctic Sun were used on a limited number of patients in our study. Lastly, our study was conducted at a single institution and involved a small number of subjects. Thus, future studies with larger cohorts are needed to confirm these findings.

\section{Conclusions}

GWR, ONSD, and LOB/SE on initial brain CT scans are associated with neurological outcome in patients who underwent ECPR. The new risk prediction model, which uses a composite of GWR, ONCD, and LOB/SE, could provide better information on neurologic outcomes in patients who underwent ECPR.

\section{Key messages}

- Brain CT may be more helpful for predicting neurological outcome in ECPR patients under sedation because some clinical signs and examinations may also be confounded by sedation.

- GWR, ONSD, and LOB/SE on initial brain CT scans are associated with neurological outcome in patients who underwent ECPR.

- A composite of GWR, LOB/SE, and ONSD was a powerful predictor of neurological outcomes compared with each parameter alone. In real-world practice, combined CT parameters could provide improved information on early neurological prognosis in patients who underwent ECPR.

\section{Additional file}

Additional file 1: Table S1. Specificity and sensitivity of $C T$ markers for poor neurological outcomes. (DOCX $21 \mathrm{~kb}$ )

\section{Abbreviations}

AUC: Area under the curve; BG: Basal ganglia; CC: Corpus callosum; Cl: Confidence interval; CN: Caudate nucleus; CPC: Cerebral Performance Categories; CPR: Cardiopulmonary resuscitation; CT: Computed tomography; ECMO: Extracorporeal membrane oxygenation; ECPR: Extracorporeal cardiopulmonary resuscitation; GWR: Gray-to-white matter ratio; GWR-AV: Average of gray-to-white matter ratios; GWR-BG: Gray-to-white matter ratio in the basal ganglia; GWR-CO: Gray-to-white matter ratio in the cortex; GWR-SI: Simplified gray-to-white matter ratio; ICU: Intensive care unit; IQR: Interquartile range; LOB: Loss of the boundary between gray matter and white matter; MC: Medial cortex; MWM: Medial white matter; ONSD: Optic nerve sheath diameter; PIC: Posterior limb of the internal capsule:

PU: Putamen; ROC: Receiver operating characteristic; ROI: Regions of interest; ROSC: Return of spontaneous circulation; SE: Cortical sulcal effacement

\section{Acknowledgements}

We appreciate the excellent statistical support of Keumhee C. Carriere, PhD, and Joonghyun Ahn, MS, at the Samsung Biomedical Research Institute.

Funding

Not applicable.

\section{Availability of data and materials}

Our data are available on the Harvard Dataverse Network (http://dx.doi.org/ 10.7910/DVN/FS7TVU) as recommended repositories of Critical Care.

\section{Authors' contributions}

JAR participated in study design, data collection, drafting of the manuscript, and the statistical analysis. CRC participated in the statistical analysis and helped draft the manuscript. YHC participated in study design, performed the statistical analysis, and drafted the manuscript. KS participated in the statistical analysis and helped draft the manuscript. GYS participated in study design and coordination and helped draft the manuscript. TKP participated in study design and drafting of the manuscript. YBS participated in study conception and design, data collection, and drafting of the manuscript. JYH participated in study design and coordination. JHC participated in the statistical analysis and helped draft the manuscript. HCG participated in the statistical analysis and helped draft the manuscript. SHC participated in the statistical analysis and helped draft the manuscript. JHY participated in study conception and design, data collection, and drafting of the manuscript. All authors read and approved the final manuscript.

\section{Competing interests}

The authors declare that they have no competing interests.

\section{Consent for publication}

Not applicable. This study did not contain any individual person's data in any form (including individual details, images, or videos).

Ethics approval and consent to participate

This study was approved by the Institutional Review Board of the Samsung Medical Center (SMC 2016-05-086) according to the Declaration of Helsinki on reviewing and publishing information from patient's records. Informed consent was waived because of the retrospective nature of the study.

\section{Author details}

${ }^{1}$ Department of Critical Care Medicine, Samsung Medical Center, Sungkyunkwan University School of Medicine, 81 Irwon-ro, Gangnam-gu, Seoul, Republic of Korea. ${ }^{2}$ Department of Thoracic and Cardiovascular Surgery, Samsung Medical Center, Sungkyunkwan University School of Medicine, 81 Irwon-ro, Gangnam-gu, Seoul, Republic of Korea. ${ }^{3}$ Division of Pulmonary and Critical Care Medicine, Department of Medicine, Samsung Medical Center, Sungkyunkwan University School of Medicine, 81 Irwon-ro, Gangnam-gu, Seoul, Korea. ${ }^{4}$ Division of Cardiology, Department of Medicine, Samsung Medical Center, Sungkyunkwan University School of Medicine, 81 Irwon-ro, Gangnam-gu, Seoul, Republic of Korea.

Received: 8 September 2016 Accepted: 4 January 2017

Published online: 25 January 2017

\section{References}

1. Nielsen N, Wetterslev J, Cronberg T, Erlinge D, Gasche Y, Hassager C, et al. Targeted temperature management at 33 degrees $C$ versus 36 degrees $C$ after cardiac arrest. N Engl J Med. 2013;369:2197-206.

2. Young GB. Clinical practice. Neurologic prognosis after cardiac arrest. N Engl J Med. 2009:361:605-11.

3. Sandroni C, Cariou A, Cavallaro F, Cronberg T, Friberg H, Hoedemaekers C, et al. Prognostication in comatose survivors of cardiac arrest: an advisory 
statement from the European Resuscitation Council and the European Society of Intensive Care Medicine. Intensive Care Med. 2014;40:1816-31.

4. Langkjaer S, Hassager C, Kjaergaard J, Salam I, Thomsen JH, Lippert FK, et al. Prognostic value of reduced discrimination and oedema on cerebral computed tomography in a daily clinical cohort of out-of-hospital cardiac arrest patients. Resuscitation. 2015:92:141-7.

5. Lee YH, Oh YT, Ahn HC, Kim HS, Han SJ, Lee JJ, et al. The prognostic value of the grey-to-white matter ratio in cardiac arrest patients treated with extracorporeal membrane oxygenation. Resuscitation. 2016;99:50-5.

6. Hirsch KG, Mlynash M, Jansen S, Persoon S, Eyngorn I, Krasnokutsky MV, et al. Prognostic value of a qualitative brain MRI scoring system after cardiac arrest. J Neuroimaging. 2015;25:430-7.

7. Naples R, Ellison E, Brady WJ. Cranial computed tomography in the resuscitated patient with cardiac arrest. Am J Emerg Med. 2009;27:63-7.

8. Inamasu J, Miyatake S, Suzuki M, Nakatsukasa M, Tomioka H, Honda M, et al Early CT signs in out-of-hospital cardiac arrest survivors: temporal profile and prognostic significance. Resuscitation. 2010;81:534-8.

9. Metter RB, Rittenberger JC, Guyette FX, Callaway CW. Association between a quantitative $C T$ scan measure of brain edema and outcome after cardiac arrest. Resuscitation. 2011;82:1180-5.

10. Kim SH, Choi SP, Park KN, Youn CS, Oh SH, Choi SM. Early brain computed tomography findings are associated with outcome in patients treated with therapeutic hypothermia after out-of-hospital cardiac arrest. Scand J Trauma Resusc Emerg Med. 2013;21:57.

11. Hwan Kim Y, Ho Lee J, Kun Hong C, Won Cho K, Hoon Yeo J, Ju Kang M, et al. Feasibility of optic nerve sheath diameter measured on initial brain computed tomography as an early neurologic outcome predictor after cardiac arrest. Acad Emerg Med. 2014;21:1121-8.

12. Morimura N, Sakamoto T, Nagao K, Asai Y, Yokota H, Tahara Y, et al. Extracorporeal cardiopulmonary resuscitation for out-of-hospital cardiac arrest: a review of the Japanese literature. Resuscitation. 2011;82:10-4.

13. Sakamoto T, Morimura N, Nagao K, Asai Y, Yokota H, Nara S, et al. Extracorporeal cardiopulmonary resuscitation versus conventional cardiopulmonary resuscitation in adults with out-of-hospital cardiac arrest: a prospective observational study. Resuscitation. 2014;85:762-8.

14. Chen Y-S, Lin J-W, Yu H-Y, Ko W-J, Jerng J-S, Chang W-T, et al. Cardiopulmonary resuscitation with assisted extracorporeal life-support versus conventional cardiopulmonary resuscitation in adults with in-hospital cardiac arrest: an observational study and propensity analysis. Lancet. 2008;372:554-61.

15. Massetti M, Tasle M, Le Page O, Deredec R, Babatasi G, Buklas D, et al. Back from irreversibility: extracorporeal life support for prolonged cardiac arrest. Ann Thorac Surg. 2005;79:178-83. discussion 183-4.

16. Megarbane B, Leprince P, Deye N, Resiere D, Guerrier G, Rettab S, et al. Emergency feasibility in medical intensive care unit of extracorporeal life support for refractory cardiac arrest. Intensive Care Med. 2007;33:758-64.

17. Nagao K, Kikushima K, Watanabe K, Tachibana E, Tominaga Y, Tada K, et al. Early induction of hypothermia during cardiac arrest improves neurological outcomes in patients with out-of-hospital cardiac arrest who undergo emergency cardiopulmonary bypass and percutaneous coronary intervention. Circ J. 2010;74:77-85.

18. Ryu JA, Cho YH, Sung $\mathrm{K}$, Choi $\mathrm{SH}$, Yang JH, Choi JH, et al. Predictors of neurological outcomes after successful extracorporeal cardiopulmonary resuscitation. BMC Anesthesiol. 2015;15:26.

19. Park SB, Yang JH, Park TK, Cho YH, Sung K, Chung CR, et al. Developing a risk prediction model for survival to discharge in cardiac arrest patients who undergo extracorporeal membrane oxygenation. Int J Cardiol. 2014;177:1031-5.

20. Maekawa K, Tanno K, Hase M, Mori K, Asai Y. Extracorporeal cardiopulmonary resuscitation for patients with out-of-hospital cardiac arrest of cardiac origin: a propensity-matched study and predictor analysis. Crit Care Med. 2013:41:1186-96.

21. Cummins RO, Chamberlain DA, Abramson NS, Allen M, Baskett PJ, Becker L, et al. Recommended guidelines for uniform reporting of data from out-ofhospital cardiac arrest: the Utstein Style. A statement for health professionals from a task force of the American Heart Association, the European Resuscitation Council, the Heart and Stroke Foundation of Canada, and the Australian Resuscitation Council. Circulation. 1991;84:960-75.

22. Kang MJ, Lee TR, Shin TG, Sim MS, Jo IJ, Song KJ, et al. Survival and neurologic outcomes of out-of-hospital cardiac arrest patients who were transferred after return of spontaneous circulation for integrated post-cardiac arrest syndrome care: another feasibility of the cardiac arrest center. J Korean Med Sci. 2014:29:1301-7.
23. Torbey MT, Selim M, Knorr J, Bigelow C, Recht L. Quantitative analysis of the loss of distinction between gray and white matter in comatose patients after cardiac arrest. Stroke. 2000;31:2163-7.

24. Legrand A, Jeanjean P, Delanghe F, Peltier J, Lecat B, Dupont H. Estimation of optic nerve sheath diameter on an initial brain computed tomography scan can contribute prognostic information in traumatic brain injury patients. Crit Care. 2013;17:R61.

25. Sekhon MS, Griesdale DE, Robba C, McGlashan N, Needham E, Walland K, et al. Optic nerve sheath diameter on computed tomography is correlated with simultaneously measured intracranial pressure in patients with severe traumatic brain injury. Intensive Care Med. 2014:40:1267-74.

26. Cummins RO, Chamberlain D, Hazinski MF, Nadkarni V, Kloeck W, Kramer E, et al. Recommended guidelines for reviewing, reporting, and conducting research on in-hospital resuscitation: the in-hospital 'Utstein style'. Circulation. 1997:95:2213-39.

27. DeLong ER, DeLong DM, Clarke-Pearson DL. Comparing the areas under two or more correlated receiver operating characteristic curves: a nonparametric approach. Biometrics. 1988;44:837-45.

28. Schisterman EF, Perkins NJ, Liu A, Bondell H. Optimal cut-point and its corresponding Youden Index to discriminate individuals using pooled blood samples. Epidemiology. 2005;16:73-81.

29. Ruopp MD, Perkins NJ, Whitcomb BW, Schisterman EF. Youden Index and optimal cut-point estimated from observations affected by a lower limit of detection. Biomed J. 2008;50:419-30.

30. Neumar RW, Shuster M, Callaway CW, Gent LM, Atkins DL, Bhanji F, et al Part 1: Executive summary: 2015 American Heart Association guidelines update for cardiopulmonary resuscitation and emergency cardiovascular care. Circulation. 2015;132:S315-67.

31. Shin TG, Choi JH, Jo IJ, Sim MS, Song HG, Jeong YK, et al. Extracorporeal cardiopulmonary resuscitation in patients with inhospital cardiac arrest: a comparison with conventional cardiopulmonary resuscitation. Crit Care Med. 2011;39:1-7.

32. Samaniego EA, Mlynash M, Caulfield AF, Eyngorn I, Wijman CA. Sedation confounds outcome prediction in cardiac arrest survivors treated with hypothermia. Neurocrit Care. 2011;15:113-9.

\section{Submit your next manuscript to BioMed Central and we will help you at every step:}

- We accept pre-submission inquiries

- Our selector tool helps you to find the most relevant journal

- We provide round the clock customer support

- Convenient online submission

- Thorough peer review

- Inclusion in PubMed and all major indexing services

- Maximum visibility for your research

Submit your manuscript at www.biomedcentral.com/submit
C) Biomed Central 